\title{
Dielectrophoretic Alignment and Pearl Chain Formation of Single-Walled Carbon Nanotubes in Deuterium Oxide Solution
}

\author{
Dong Su Lee ${ }^{1, \star}$ and Yung Woo Park ${ }^{2}$ \\ ${ }^{1}$ Institute of Advanced Composite Materials, Korea Institute of Science and Technology, Jeonbuk 565-902, Korea \\ ${ }^{2}$ Department of Physics and Astronomy, Seoul National University, Seoul 151-747, Korea
}

\author{
Article Info \\ Received 1 August 2012 \\ Accepted 25 September 2012 \\ *Corresponding Author \\ E-mail: d.s.lee@kist.re.kr \\ Tel: $+82-63-710-7576$

\section{Open Access} \\ DOI: http://dx.doi.org/ \\ 10.5714/CL.2012.13.4.248 \\ This is an Open Access article distributed \\ under the terms of the Creative Commons \\ Attribution Non-Commercial License \\ (http://creativecom mons.org/licenses/ \\ by-nc/3.0/) which permits unrestricted \\ non-commercial use, distribution, and \\ reproduction in any medium, provided \\ the original work is properly cited.
}

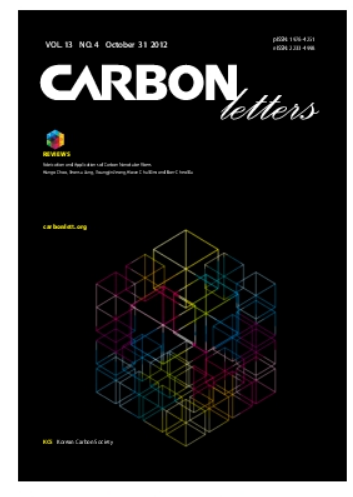

http://carbonlett.org

pISSN: 1976-4251

elSSN: 2233-4998

Copyright $\odot$ Korean Carbon Society

\begin{abstract}
Dielectrophoretic filtering and alignment of single-walled carbon nanotubes (SWCNTs) were tested using deuterium oxide as a solvent. A solution of deuterium oxide-SWCNTs was dropped on top of a silicon chip and an ac electric field was applied between pre-defined electrodes. Deuterium oxide was found to be a better solvent than hydrogen oxide for the dielectrophoresis process with higher efficiency of filtering. This was demonstrated by comparing Raman spectra measured on the initial solution with those measured on the filtered solution. We found that the aligned nanotubes along the electric field were not deposited on the substrate but suspended in solution, forming chain-like structures along the field lines. This so-called pearl chain formation of CNTs was verified by electrical measurements through the aligned tubes. The solution was frozen in liquid nitrogen prior to the electrical measurements to maintain the chain formation. The current-voltage characteristics for the sample dem onstrate the existence of conduction channels in the solution, which are associated with the SWCNT chain structures
\end{abstract}

Key words: carbon nanotubes, dielectrophoresis, dielectrophoretic filterings, pearl chain formation

\section{Introduction}

Single-walled carbon nanotubes (SWCNTs) are one-dimensional carbon-based nanom aterials, and are considered a promising candidate for future electronic applications due to their unique electronic properties [1-6]. For instance, ballistic transport with high carrier mobility can be achieved. Notably, SWCNTs can carry high current density exceeding $10^{9} \mathrm{~A} / \mathrm{cm}^{2}$ [7]. Such excellent electrical properties stem from the relativistic nature of particles in SWCNTs similar to those in graphene [8,9], which can be thought of as an unrolled basic structure of a SWCNT. However, graphene is not suitable for application to field effect transistors, because it has only a zero band gap and the achievable on/off ratio is therefore little more than one order of magnitude. Recently, a high on/off ratio of $10^{5}$ has been realized in a graphene-silicon Schottky barrier [10]. However, these barristors are not based on the intrinsic graphene properties, and thus it is not yet possible to realize the concept of "all carbon electronics", which is often cited as a potential replacement for current silicon-based electronics [11]. On the other hand, SWCNTs exhibit either a semiconducting or metallic character depending on their chirality and, moreover, the band gap of semiconducting tubes can be tuned by controlling the chirality [3]. A high on/off ratio of the source-drain current through the field effect transistor devices using semiconducting SWCNT channels (more than $10^{\circ}$ ) has been achieved $[5,7,12,13]$. Also, SWCNT crossed junction transistors with a very short channel length close to tube diameter have shown fast switching frequency of around 200 $\mathrm{GHz}[14,15]$. Despite the excellent properties of SWCNTs as field effect transistor channel materials, it is not yet possible to obtain only semiconducting tubes from the growth stage Instead, separation of the semiconducting tubes from bulk materials appears to be a more 
feasible route. Researchers have attempted to separate semiconducting SWCNTs by various approaches, such as centrifugationbased separation using the difference of the surfactant stabilization [16], repeated dielectrophoretic filtering [17], DNA-assisted separation [18], selective removal of metallic SWCNTs with small diameters by using nitric and sulfuric acid [19], and, very recently, single-chirality separation by gel chromatography [20]. Dielectrophoretic filtering is a chemical-free and nondestructive method, as the separation process is based only on the differences in the physical properties and dielectric constants of semiconducting and metallic tubes. However, the mechanism of the separation process is not yet clear. For instance, even in the region where the separation of electrodes is very large $(\sim 100 \mu \mathrm{m})$ compared to the tube length, the metallic tubes are captured by the applied ac electric field between the electrodes. This behavior cannot be explained solely by the dielectrophoretic force induced by an external electric field

Here, we show that metallic SWCNTs aligned by dielectrophoresis are not deposited directly onto the substrate during the process but remain suspended in a solution due to the effect of pearl chain formation [21-24]. This was demonstrated by an optical microscopy study as well as by electrical measurements through the SWCNTs chains formed inside the solution

\section{Experimental}

\subsection{SWCNTs solution in deuterium oxide}

The sample was prepared by suspending SWCNTs in deuterium oxide containing $4 \mathrm{wt} \%$ of sodium dodecyl sulfate (SDS) as a surfactant. We used SWCNTs synthesized by a high pressure deposition carbon monoxide method in the experiment [25]. After $10 \mathrm{~min}$ of ultrasonic agitation, the sample was centrifuged at $50000 \mathrm{~g}$ for $30 \mathrm{~min}$ and the upper $80 \%$ of the solution was carefully taken. The centrifugation process is required for extracting SWCNT bundles. The specific gravity of an individual SWCNT wrapped with SDS (SWCNT-SDS) is around $1.0 \mathrm{~g} / \mathrm{cm}^{3}$ and that of a bundle of several SWCNTs wrapped with SDS (bundleSDS) is around $1.2 \mathrm{~g} / \mathrm{cm}^{3}$. Deuterium oxide was used instead of hydrogen oxide for better extraction of bundles because it has a specific gravity $\left(1.1 \mathrm{~g} / \mathrm{cm}^{3}\right)$ larger than that of SWCNT-SDS and smaller than that of bundle-SDS [26]. The taken suspension showed no aggregation for more than a week.

\subsection{Electrodes structure for dielectrophoresis}

Wheel-type electrode structures were fabricated on top of a $\mathrm{Si}$ chip covered with a $500 \mathrm{~nm}$ thick thermal oxide using electron beam lithography and electron beam evaporation of $\mathrm{Ti} / \mathrm{Au}(5$ $\mathrm{nm} / 100 \mathrm{~nm}$ ). Here, Ti serves as an adhesion layer. The electrode configuration is the same as that used in Lee et al. [17]. The electric field applied to the electrodes generates not only a vertical gradient but also a horizontal gradient of the electric field. This chip was used for an in situ Raman measurements, which were performed while an ac electric field was applied. Another type of electrode structure with a two-probe configuration was fabricated by the same procedure. The width of the gap was $35 \mu \mathrm{m}$ and the distance between the electrodes was $6 \mu \mathrm{m}$. The electrodes were used for electrical measurements through the SWCNT chain.

\subsection{Raman spectroscopy}

Raman spectroscopy was used to determine the metallic and semiconducting SWCNTs contents in a solution depending on the strength of the applied electric field [17,27]. An $\mathrm{Ar}+$ ion laser with a wavelength of $514.5 \mathrm{~nm}$ (excitation energy of $2.41 \mathrm{eV}$ ) was used for the incident light source. The laser spot size was around $3 \mu \mathrm{m}$ in diameter. Raman spectra were obtained either for the suspension in the deuterium oxide solution or for SWCNTs deposited on the $\mathrm{SiO}_{2}$ substrate after deuterium oxide was evaporated.

\section{Results and Discussion}

\subsection{In situ Raman spectra on precipitate and supernatant SWCNTs during the dielectropho- resis process}

In a previous work [17], we extracted semiconducting SWCNTs in a hydrogen oxide solution using repeated dielectrophoretic filtering. A schematic illustration of the dielectrophoresis process is depicted in Fig. 1. When a uniform electric field is applied to SWCNTs suspended in a solution, the generated torque due to the dipole moment of the tubes aligns the SWCNTs in the direction of the applied field. The force is, however, balanced as long as the SWCNTs are not charged. No translational motion of the tubes is expected in this case. However, when the applied field is not uniform, the aligned SWCNTs that are suspended in the solution are attracted or repelled in the direction of the electrical field gradient. This dielectrophoretic force vector is given by $[23,28]$,

$$
\begin{aligned}
& \vec{F}_{D E P}=\frac{\pi d^{2} l}{4} \varepsilon_{m} \operatorname{Re}\left(\frac{\varepsilon_{t}^{*}-\varepsilon_{m}^{*}}{\varepsilon_{m}^{*}+\left(\varepsilon_{t}^{*}-\varepsilon_{m}^{*}\right) L}\right)|\vec{E}| \nabla|\vec{E}|, \varepsilon_{t, m}^{*} \\
& =\varepsilon_{t, m}-i \frac{\sigma_{i, m}}{\omega} .
\end{aligned}
$$

Here, $\mathrm{d}$ and $\mathrm{l}$ denote the diameter and the length of the tube, respectively, $\vec{E}$ is the external electric field, $\varepsilon_{f}^{*}\left(\varepsilon_{m}^{*}\right)$ is the com-

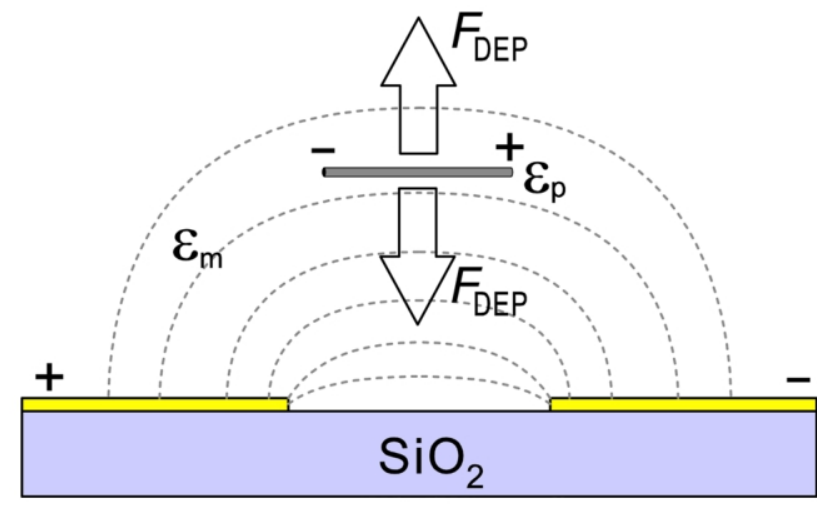

Fig. 1. Schematic drawing of the dielectrophoresis process. The illustration shows a side view of the Si chip with a pair of electrodes to apply an electric field and also shows a tube aligned along the applied field. The applied dielectrophoretic force is either downwards or upwards depending on the dielectric constant of the single walled nanotube. 


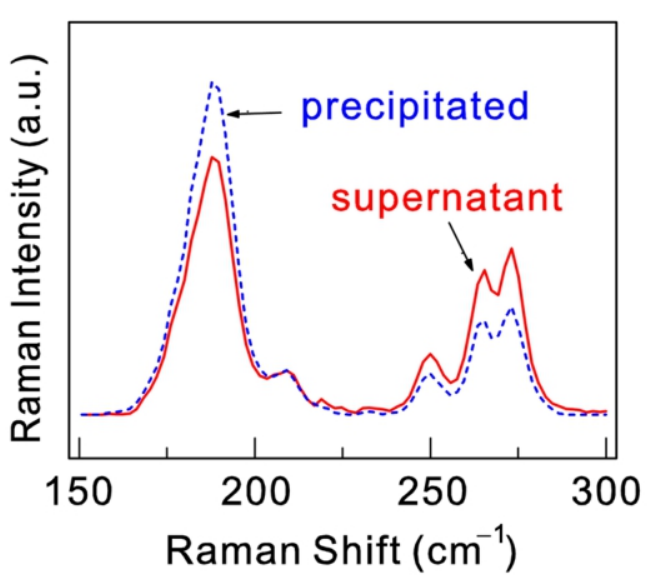

(a)

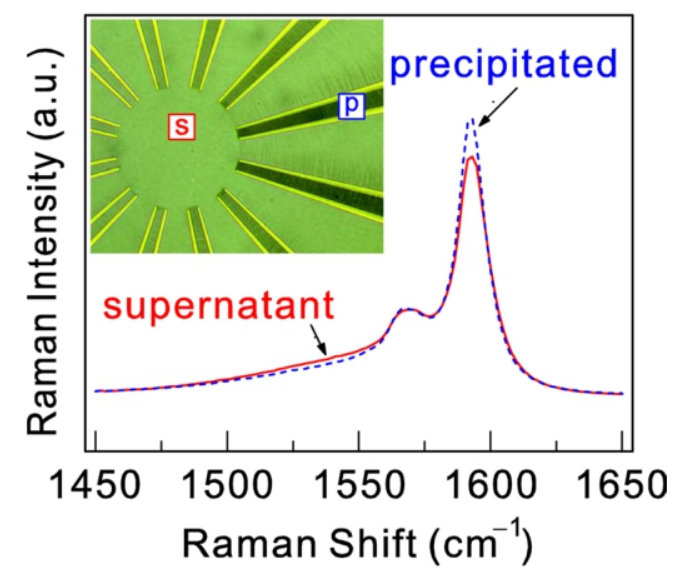

(b)

Fig. 2. In situ Raman spectra of the single walled nanotubes (SWCNTs) aligned between the electrodes (red solid lines) and spectra measured at a position away from the aligned SWCNTs (blue dashed lines) at $150-300 \mathrm{~cm}^{-1}$ (a) and at $1450-1650 \mathrm{~cm}^{-1}$ (b). The measurements were carried out while an ac electric field was applied for all the electrode pairs. Inset in (b) shows an optical microscope image of the deuterium oxide-SWCNTs solution on the wheel-type electrodes. The Raman spectra for the precipitated and supernatant SWCNTs were obtained at the positions marked by "p" and "s," respectively.

plex dielectric constant of a tube (medium), $\sigma_{t}\left(\sigma_{m}\right)$ is the conductivity of the tube (medium), and $\omega$ is the angular frequency of the ac electric field. The depolarization factor $\mathrm{L}$ is of the order of $10^{-5}$ for HiPco tubes [28]. In the parallel electrode geometry shown in Fig. 1, the direction of the field gradient $(\nabla|\vec{E}|$ is down to the substrate. The conductivity of the medium varies with the surfactant concentration. However, the conductivity and dielectric constant are many orders of magnitude larger than those of hydrogen oxide or deuterium oxide, even for high surfactant concentrations. The dielectric constant of hydrogen oxide or deuterium oxide can be assumed as $\varepsilon_{\mathrm{m}} \sim 80 \varepsilon_{0}$, where $\varepsilon_{0}$ is the dielectric constant of vacuum. According to Eq. (1), the sign of the dielectrophoretic force is always positive for any frequency [29]. Metallic SWCNTs are therefore pulled down to the substrate, where the electric field is the strongest. For semiconducting SWCNTs, which have a dielectric constant of $\varepsilon_{\mathrm{m}} \sim 2-5 \varepsilon_{0}$ $[29,30]$, the sign of the dielectrophoretic force is dependent on the frequency of the applied ac field as well as on the surfactant concentration. In our experiment conditions the dielectrophoretic force for semiconducting SWCNTs is negative and the SWCNTs remain in the solution [17].

Fig. 2 shows the in situ Raman spectra around the radial breathing mode (RBM) and around the $\mathrm{G}$ mode for supernatant and precipitated SWCNTs. The spectra were obtained as follows. A small amount of the SWCNTs-deuterium oxide solution was dropped on top of the substrate with wheel-type electrode structures. An ac voltage with a peak-to-peak amplitude of $10 \mathrm{~V}$ at a frequency of $13 \mathrm{MHz}$ was then applied between the wheeltype electrode pairs using a function generator. The inset of Fig. $2 \mathrm{~b}$ shows an optical microscopy image taken during application of the electric field. Between the electrodes, SWCNTs are accumulated due to dielectrophoretic force. The Raman spectra were then obtained on the aligned SWCNTs between the electrodes (precipitated) and on the region where no electric field is applied, i.e. in the center of the wheel-type electrodes (supernatant). The regions are marked in the optical image. Generally, the $\mathrm{RBM}$ frequencies observed in a resonant Raman measurement are from only several species of the tube, the optical transition energy of which equals the incident laser energy [8]. According to the plot of the tube diameter versus the allowed optical transition energy, known as a "Kataura plot" [31], when using a $514.5 \mathrm{~nm}$ laser, the RBM peaks from semiconducting tubes appear at $130-200 \mathrm{~cm}^{-1}$ and the peaks from metallic tubes at 220$280 \mathrm{~cm}^{-1}$. In our data shown in Fig. 2a, the peak at around 190 $\mathrm{cm}^{-1}$ represents semiconducting SWCNTs. The intensity of this peak in the spectrum obtained in the supernatant region is larger than the intensity obtained in the precipitated region. Metallic peaks in the range from 230 to $280 \mathrm{~cm}^{-1}$ are, on the other hand, larger in the precipitated region. These results imply that the ac electric field captures metallic SWCNTs between the electrodes, leaving semiconducting SWCNTs in the solution. The Raman G mode is a superposition of semiconducting and metallic tubes Two sharp peaks from around $1560-1593 \mathrm{~cm}^{-1}$ are induced by semiconducting SWCNTs and a broad and asymmetric peak at around $1540 \mathrm{~cm}^{-1}$ is induced by the metallic SWCNTs $[32,33]$. In Fig. $2 b$, the slightly smaller intensity of the sharp semiconducting peak at around $1600 \mathrm{~cm}^{-1}$ for the precipitated region also shows the dielectrophoretic effect.

\subsection{Dielectrophoretic filtering using deute- rium oxide solution}

We performed dielectrophoretic filtering for the deuterium oxide solution. The details of the process are the same as in a previous work using a hydrogen oxide solution [17]. With the use of deuterium oxide as a solvent instead of hydrogen oxide the filtration efficiency was improved. Fig. 3 displays the Raman spectra on the initial deuterium oxide solution and the filtered sample. For this measurement, the SWCNTs were deposited on $\mathrm{SiO}_{2}$ substrates and the deuterium oxide was dried before the Raman measurements. The fraction of semiconducting SWCNTs among all the remaining SWCNTs in the filtered solution was estimated to be around $86 \%$ using an analysis of the peak intensity change. This shows that the filtration efficiency is significantly improved when using deuterium oxide, given that this level of semiconducting SWCNT content could only 


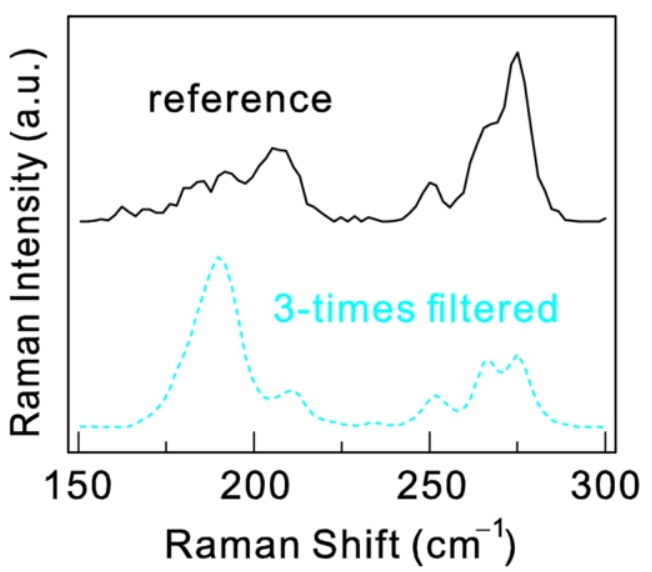

(a)

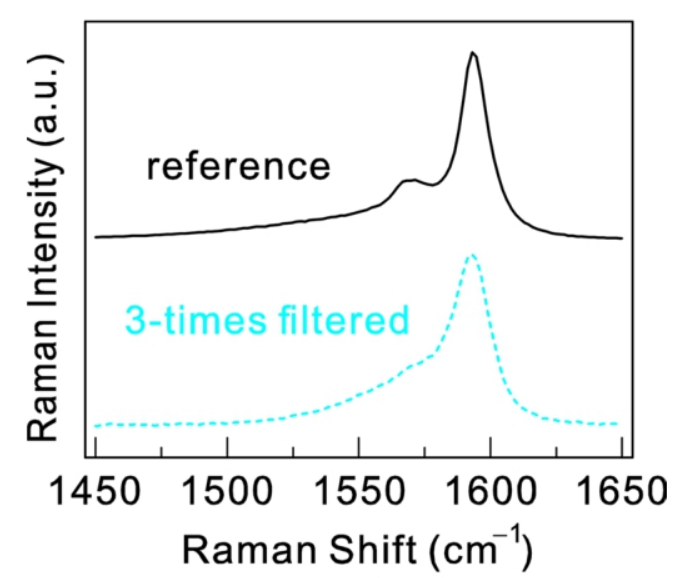

(b)

Fig. 3. Raman spectra of the sample filtered three times together with spectra of the pristine deuterium oxide-single walled nanotubes solution for comparison at $150-300 \mathrm{~cm}^{-1}$ (a) and at $1450-1650 \mathrm{~cm}^{-1}$ (b).

be achieved with at least 3 repeated filtering steps when using a hydrogen oxide solution [17].

\subsection{Pearl chain formation}

We note that the SWCNTs are accumulated even in the region where the distance between the electrodes is more than $100 \mu \mathrm{m}$. The behavior cannot be understood only by dielectrophoretic force, since the electrode spacing is much larger than the tube length and, moreover, the electric field is very small at this region compared to the field near the center of the chip. We also found that the aggregated SWCNTs between the electrodes dispersed into the solution again as soon as the electric field was turned off. These phenomena can be explained by a pearl chain formation [21-24]. A conceptual diagram of the pearl chain formation of the SWCNTs is shown in Fig. 4. In general, even under an external uniform electric field, the local electric field will bend near a dielectric particle. This self-induced nonuniform electric field near the particle will attract another dielectric particle by dielectrophoretic force, which occurs locally. As a result, the particles suspended in a solution will be linked to each other, forming a chain-like structure along the field lines. In our dielec-

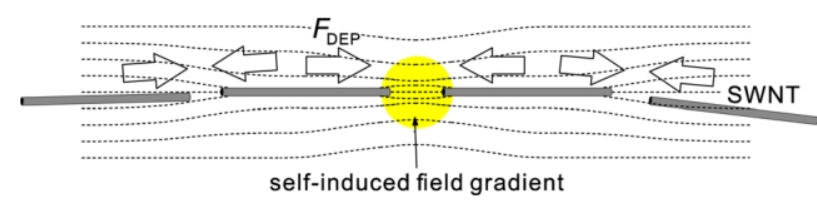

Fig. 4. Schematic drawing of pearl chain formation of single walled nanotubes (SWCNTs) in a solution. Dashed lines show the field lines and the arrows indicate the dielectrophoretic force due to the self-induced electric field gradient.

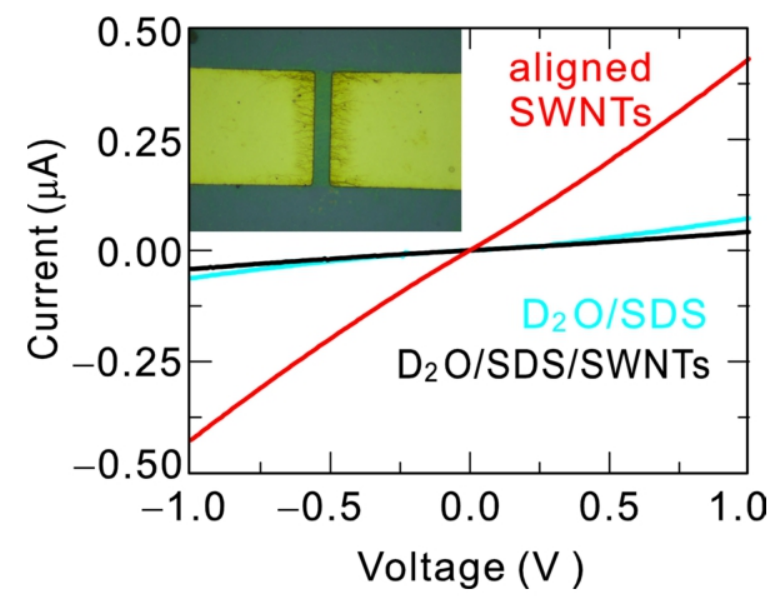

Fig. 5. I-V characteristics of the single walled nanotubes (SWCNTs) chains formed by the application of the electric field together with characteristics of the samples of the deuterium oxide/sodium dodecyl sulfate (SDS) solution and SWCNTs dispersed in the solution without dielectrophoresis alignment for comparison. All the measurements were done in liquid nitrogen. Details can be found in the text. Inset shows an optical microscope image of the electrodes used for this experiment. SWCNTs are aligned by the electric field. The gap between the electrodes is $6 \mu \mathrm{m}$.

trophoresis experiment, the tube-tube dielectrophoretic interaction may be stronger than the dielectrophoretic force induced by the external field gradient. The aligned tubes are therefore not deposited on the substrate but instead are suspended in the solution, thereby maintaining their chain formation.

\subsection{Current-voltage characteristics through SWCNT chains}

The pearl chain formation of aligned SWCNTs by the external electric field was dem onstrated by an electrical measurement between the electrodes that had been used for the application of ac electric field. We fabricated simpler electrode structures with two wide electrodes, as shown in the inset of Fig. 5. The distance between the electrodes is $6 \mu \mathrm{m}$ and the width of the electrodes is $35 \mu \mathrm{m}$. In the image, aligned tubes are visible since the image was obtained after the dielectrophoretic alignment. In order to maintain the SWCNT chains we put the chip into liquid nitrogen during application of the electric field. The deuterium oxide solution was then frozen immediately and the SWCNT chains were fixed in the solidified solution. The electric field was turned off before electrical measurements. At the liquid nitrogen temperature (around $77 \mathrm{~K}$ ), we measured current-voltage (I-V) characteristics through the SWCNT chains using the electrodes that had been used for dielectrophoresis. We swept the voltage between the electrodes and measured the current through the 
chains. We also prepared two more samples by freezing the SWCNTs-deuterium oxide solution on a chip without applying an electric field and by freezing only the deuterium water solution containing only SDS for comparison. The results are shown in Fig. 5. The $I-V$ characteristics are linear up to $1 \mathrm{~V}$ for all the samples. The $I-V$ characteristics for SWCNTs chains show a much higher conductivity with a resistance that is $2 \mathrm{M} \Omega$ less than that of the other samples. Current leakage was observed and the leakage level was similar for both the the deuterium water solution containing only SDS and the SWCNTs-deuterium oxide solution. This implies that the leakage is due to ionic conduction in the deuterium oxide solution without any contribution of SWCNTs. Thus, we can conclude that the SWCNTs are randomly dispersed and do not make a conduction path in the solution. Despite the leakage current, it is clear that the conduction channel for the aligned sample is the SWCNTs chains formed by the applied ac electric field, since the leakage current is around 1 order of magnitude smaller than the current through the chain

\section{Conclusions}

We tested dielectrophoretic filtering for SWCNTs dispersed in a deuterium oxide solution. The results demonstrate that the deuterium oxide solution shows better efficiency for dielectrophoretic separation of semiconducting and metallic SWCNTs. We found that a large amount of the tubes aligned along the applied electric field are not deposited on the substrate but remained suspended in the deuterium oxide solution. The aggregation of aligned SWCNTs is released when the electric field is turned off. We suggest that the SWCNTs not only are aligned by the external electric field but also dielectrophoretically interact with the neighboring tubes due to the self-induced electric field gradient near the tubes. The SWCNTs are consequently attracted to each other, forming chain-like structures. A pearl chain formation of SWCNTs was demonstrated by electrical measurement through the chains of SWCNTs in liquid nitrogen.

\section{Acknowledgements}

This work was supported by a grant from the Korea Institute of Science and Technology (KIST) institutional program.

\section{References}

[1] Iijima S. Helical microtubules of graphitic carbon. Nature, 354, 59 (1991). http://dx. doi.org/doi: 10.1038/354056a0

[2] Dekker C. Carbon nanotubes as molecular quantum wires. Physics Today, 52, 22 (1999). http://dx. doi. org/10. 1063/1.882658

[3] Dresselhaus MS, Dresselhaus G, Avouris P. Carbon nanotubes synthesis, structural properties and applications, Springer, New York (2001)

[4] Tans SJ, Verschueren ARM, Dekker C. Room-temperature transistor based on a single carbon nanotube. Nature, 393, 49 (1998) http://dx.doi.org/10.1038/29954

[5] Derycke V, Martel R, Appenzeller J, Avouris P. Controlling doping and carrier injection in carbon nanotube transistors. Appl Phys
Lett, 80, 2773 (2002). http://dx. doi. org/10.1063/1.1467702.

[6] Nygard J, Cobden DH, Bockrath M, McEuen PL, Lindelof PE Electrical transport measurements on single-walled carbon nanotubes. Appl Phys A: Mater Sci Process, 69, 297 (1999). http:// dx. doi. org/10.1007/s003390051004.

[7] Yao Z, Kane CL, Dekker C. High-field electrical transport in single-wall carbon nanotubes. Phys Rev Lett, 84, 2941 (2000). http:/ dx. doi. org/10.1103/PhysRevLett.84.2941

[8] Novoselov KS, Geim AK, Morozov SV, Jiang D, Katsnelson MI, Grigorieva IV, Dubonos SV, Firsov AA. Two-dimensional gas of massless Dirac fermions in graphene. Nature, 438, 197 (2005) http://dx.doi.org/10.1038/nature04233.

[9] Zhang YB, Tan YW, Stormer HL, Kim P. Experimental observation of the quantum Hall effect and Berry's phase in graphene. Nature, 438, 201 (2005). http://dx. doi.org/10.1038/nature04235

[10] Yang H, Heo J, Park S, Song HJ, Seo DH, Byun KE, Kim P, Yoo I, Chung HJ, Kim K. Graphene barristor, a triode device with a gate-controlled schottky barrier. Science, 336, 1140 (2012). http:/ dx. doi.org/10.1126/science. 1220527

[11] Avouris P, Chen ZH, Perebeinos V. Carbon-based electronics Nat Nanotechnol, 2, 605 (2007). http://dx. doi.org/10.1038/nnano. 2007.300

[12] Tans SJ, Devoret MH, Dai HJ, Thess A, Smalley RE, Geerligs LJ, Dekker C. Individual single-wall carbon nanotubes as quantum wires. Nature, 386, 474 (1997). http://dx.doi org $/ 10.1038 / 386474 \mathrm{a} 0$

[13] Lin YM, Appenzeller J, Avouris P. Ambipolar-to-unipolar conversion of carbon nanotube transistors by gate structure engineering. Nano Lett, 4, 947 (2004). http://dx.doi.org/10.1021/nl049745j

[14] Lee DS, Svensson J, Lee SW, Park YW, Campbell EEB. Fabrication of crossed junctions of semiconducting and metallic carbon nanotubes: a CNT-gated CNT-FET. J Nanosci Nanotechnol, 6, 1325 (2006). http://dx.doi.org/10.1166/jnn.2006.321.

[15] Svensson J, Tarakanov Y, Lee DS, Kinaret JM, Park YW, Campbell EEB. A carbon nanotube gated carbon nanotube transistor with 5 ps gate delay. Nanotechnology, 19, (2008). http://dx. doi org/10.1088/0957-4484/19/32/325201.

[16] Haddon RC, Sippel J, Rinzler AG, Papadimitrakopoulos F. Purification and separation of carbon nanotubes. MRS Bull, 29, 252 (2004). http://dx.doi.org/10.1557/mrs2004.76

[17] Lee DS, Kim DW, Kim HS, Lee SW, Jhang SH, Park YW, Campbell EEB. Extraction of semiconducting CNTs by repeated dielectrophoretic filtering. Appl Phys A: Mater Sci Process, 80, 5 (2005) http://dx.doi.org/10.1007/s00339-004-2992-4

[18] Zheng M, Jagota A, Semke ED, Diner BA, McLean RS, Lustig SR, Richardson RE, Tassi NG. DNA-assisted dispersion and separation of carbon nanotubes. Nat Mater, 2, 338 (2003). http://dx. doi org/10.1038/nmat877.

[19] Yang CM, Park JS, An KH, Lim SC, Seo K, Kim B, Park KA, Han S, Park CY, Lee YH. Selective removal of metallic singlewalled carbon nanotubes with small diameters by using nitric and sulfuric acids. J Phys Chem B, 109, 19242 (2005). http://dx. doi org/10.1021/jp053245c

[20] Liu HP, Nishide D, Tanaka T, Kataura H. Large-scale single-chirality separation of single-wall carbon nanotubes by simple gel chromatography. Nat Commun, 2, (2011). http://dx. doi.org/10.1038/ ncomms 1313

[21] Bezryadin A, Dekker C, Schmid G. Electrostatic trapping of single conducting nanoparticles between nanoelectrodes. Appl Phys Lett, 
71, 1273 (1997). http://dx. doi.org/10.1063/1.119871.

[22] Amlani I, Rawlett AM, Nagahara LA, Tsui RK. An approach to transport measurements of electronic molecules. Appl Phys Lett, 80, 2761 (2002). http://dx. doi.org/10.1063/1.1469655

[23] Pohl HA. Dielectrophoresis: the behavior of neutral matter in nonuniform electric fields, Cambridge University Press, Cambridge (1978).

[24] Hermanson KD, Lumsdon SO, Williams JP, Kaler EW, Velev OD Dielectrophoretic assembly of electrically functional microwires from nanoparticle suspensions. Science, 294, 1082 (2001). http:// dx.doi.org/10.1126/science.1063821

[25] Bronikowski MJ, Willis PA, Colbert DT, Smith KA, Smalley RE. Gas-phase production of carbon single-walled nanotubes from carbon monoxide via the HiPco process: a parametric study. J Vac Sci Technol A, 19, 1800 (2001). http://dx. doi.org/10.1116/1.1380721.

[26] O'Connell MJ, Bachilo SM, Huffman CB, Moore VC, Strano MS, Haroz EH, Rialon KL, Boul PJ, Noon WH, Kittrell C, Ma JP, Hauge RH, Weisman RB, Smalley RE. Band gap fluorescence from individual single-walled carbon nanotubes. Science, 297, 593 (2002). http://dx. doi.org/10.1126/science.1072631.

[27] Krupke R, Hennrich F, von Lohneysen H, Kappes MM. Separation of metallic from semiconducting single-walled carbon nanotubes. Science, 301, 344 (2003). http://dx. doi. org/10.1126/sci- ence. 1086534

[28] Krupke R, Hennrich F, Kappes MM, Lohneysen HV. Surface conductance induced dielectrophoresis of semiconducting singlewalled carbon nanotubes. Nano Lett, 4, 1395 (2004). http://dx. doi. org/10.1021/nl0493794.

[29] Benedict LX, Louie SG, Cohen ML. Static polarizabilities of single-wall carbon nanotubes. Phys Rev B, 52, 8541 (1995). http:// dx. doi. org/10.1103/PhysRevB.52.8541

[30] Ding JW, Yan XH, Cao JX. Analytical relation of band gaps to both chirality and diameter of single-wall carbon nanotubes. Phys Rev B, 66, (2002). http://dx doi. org/10.1103/PhysRevB.66.073401

[31] Kataura H, Kumazawa Y, Maniwa Y, Umezu I, Suzuki S, Ohtsuka Y, Achiba Y. Optical properties of single-wall carbon nanotubes. Synth Met, 103, 2555 (1999). http://dx. doi.org/10.1016/s03796779(98)00278-1.

[32] Yu ZH, Brus LE. (n, m) structural assignments and chirality dependence in single-wall carbon nanotube Raman scattering. J Phys Chem B, 105, 6831 (2001). http://dx. doi.org/10.1021/jp010853t.

[33] Dresselhaus MS, Dresselhaus G, Jorio A, Souza AG, Saito R Raman spectroscopy on isolated single wall carbon nanotubes. Carbon, 40, 2043 (2002). http://dx.doi.org/10.1016/s0008-6223 (02)00066-0. 